\title{
Evaluation of the plasma hydrogen isotope content by residual gas analysis at JET and AUG
}

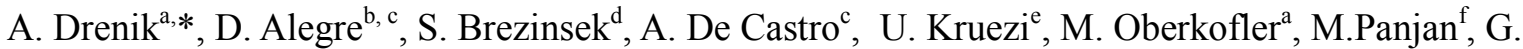 \\ Primc $^{f}$, T Reichbauer ${ }^{\mathrm{a}}$, M. Resnik ${ }^{\mathrm{f}}$, V. Rohde ${ }^{\mathrm{a}}$, M. Seibt ${ }^{\mathrm{a}}$, P. A. Schneider ${ }^{\mathrm{a}}$, T. Wauters ${ }^{\mathrm{g}}$, R. Zaplotnik ${ }^{\mathrm{f}}$, the \\ ASDEX-Upgrade and EUROfusion MST1* teams, and JET contributors** \\ ${ }^{a}$ Max-Planck-Institut für Plasmaphysik, 85748 Garching b. München, Germany \\ ${ }^{b}$ Departamento de Ingeniería Energética, UNED, C/ Juan del Rosal 12, 28040 Madrid, Spain. \\ ${ }^{c}$ Laboratorio Nacional de Fusión, CIEMAT, Avda Complutense 40, 28040 Madrid, Spain \\ ${ }^{d}$ Forschungszentrum Juelich GmbH, EURATOM Association, 52425 Juelich, Germany \\ ${ }^{e}$ Culham Centre for Fusion Energy, Culham Science Centre, Abingdon, OX14 3DB, UK,

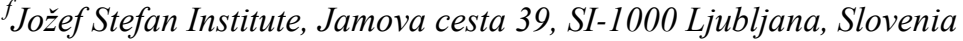 \\ ${ }^{g}$ Laboratory for Plasmaphysics, LPP-ERM/KMS, Brussels, Belgium, TEC Parner \\ * See the author list of "Overview of progress in European Medium Sized Tokamaks towards an integrated plasma- \\ edge/wall solution" by H. Meyer et al., to be published in Nuclear Fusion Special issue: Overview and Summary \\ Reports from the 26th Fusion Energy Conference (Kyoto, Japan, 17-22 October 2016) \\ ** See the author list of "Overview of the JET results in support to ITER" by X. Litaudon et al. to be published in \\ Nuclear Fusion Special issue: overview and summary reports from the 26th Fusion Energy Conference (Kyoto, \\ Japan, 17-22 October 2016)
}

\begin{abstract}
The isotope content of the plasma reflects on the dynamics of isotope changeover experiments, efficiency of wall conditioning and the performance of a fusion device in the active phase of operation. The assessment of the isotope ratio of hydrogen and methane molecules is used as a novel method of assessing the plasma isotope ratios at JET and AUG. The isotope ratios of both molecules in general shows similar trends as the isotope ratio detected by other diagnostics. At JET, the absolute values of RGA signals are in relatively good agreement with each other and with spectroscopy data, while at AUG the deviation from neutral particle analyser data are larger, and the results show a consistent spatial distribution of the isotope ratio. It is further shown that he isotope ratio of the hydrogen molecule can be used to study the degree of dissociation of the injected gas during changeover experiments.
\end{abstract}

\section{Introduction}

Monitoring the isotope content of the plasma fuel provides essential information about the operation of a fusion device. In non-active phases of operation of fusion devices, the plasmas mostly fuelled with only one hydrogen isotope at once. Between different isotope campaigns, isotope changeover experiments phases offer the opportunity to study fuel retention and release(1), and to assess the efficiency of wall conditioning techniques. In such experiments, insitu measuring of the isotope ratio of the plasma fuel provides real time information about the evolution of the processes in the reactor vessel, and is complementary to the ex-situ gas balance analysis. The methods of fuel removal, and the detection thereof, will be very soon put to the test in JET, where a pure tritium experimental (TT) campaign is planned. The TT campaign will be preceded with a hydrogen $(\mathrm{H})$ campaign, during which the deuterium $(\mathrm{D})$ inventory, accessible to the plasma, will have to be reduced sufficiently to keep the plasma D content below $1 \%$. Any leftover D is likely to produce fast neutrons through the fusion reaction which will lead to the premature filling up of the JET neutron budget. 
In the future DT campaigns at JET, and in active and especially powerplant-type fusion devices in the future, monitoring the isotope content of the fuel mixture in real-time will be critical to the control of the operation. While presently operating fusion devices are experimentally focused and thus offer many diagnostic possibilities to measure the isotope ratio, such as spectroscopic measurements(2,3) and analysis of energetic neutral particles(4), the range of diagnostics available to use in future reactors will be considerably smaller. Due to the need to ensure tritium self-sufficiency, the vessel in the future fusion devices will be surrounded by the breeding blanket, which will severely limit the access to the plasma.

Residual gas analysis (RGA) has already been shown to provide information about certain plasma-wall interaction processes in fusion devices $(5,6)$. As it does not require direct access to the plasma, nor immediate vicinity to the plasma containing vessel, it is a suitable candidate for use in future, diagnostic-limited fusion devices. Moreover, for the requirements of DT operation at Iter and JET, manufacturers now provide the option of connecting the electronic control units to the mass spectrometers by cables of several meters of length. This allows for further shielding the neutron-sensitive electronics and undisturbed operation in the DT phases. RGA is routinely utilized in dedicated isotope exchange experiments to measure the isotope ratios of the hydrogen species(7). In this contribution, we present the first campaign-wide study of the isotope ratio by RGA at JET, and at AUG in a more limited dataset. Furthermore, we extend the analysis to include the methane molecule, beside the hydrogen species.

\section{Experimental}

The RGA data, presented in this work, was acquired over hydrogen campaigns at JET and ASDEX-Upgrade (AUG). At JET, the hydrogen campaign lasted for six weeks, providing 433 pulses with good quality RGA data. This comprises of various isotope studies (L-H transition, confinement, detachment, ICRH heating, etc.) and vastly varying $\mathrm{D}_{2}$ and $\mathrm{H}_{2}$ injection schemes. Owing to a history of DT operation, the $\mathrm{H} /(\mathrm{H}+\mathrm{D})$ ratio is standardly measured with a subdivertor Penning gauge(3) and visible spectroscopy of the $\mathrm{H}$-alpha line(2). The hydrogen campaign at AUG was executed within a single day, and the resulting dataset is accordingly significantly narrower, comprising 11 plasma pulses that provided good RGA data. Among those, 7 pulses also feature $\mathrm{H} /(\mathrm{H}+\mathrm{D})$ ratios obtained with the neutral particle analyser (NPA)(4).

For the acquisition of good quality data from the discharge phase, a RGA set up must provide magnetic shielding for the mass spectrometer (MS) and differential pumping with a suitable pressure reduction. At JET, this is provided by a sub-divertor neutral gas diagnostic clusters(8). During the most recent hydrogen campaign, the RGA data was acquired by a single Hiden Analytical HAL 201 RC MS. The device was setup to record masses related to the hydrogen molecule range ( $2-4$ and 6 AMU) as well as the impurities (15 - 20 AMU, 28 and 32 AMU), with a scanning time of roughly 1.3 seconds for the whole mass range (roughly 0.09 s per mass). Although $\mathrm{D}_{2}$ also has a peak in the mass spectra at 6 AMU, only the peaks at 2, 3 and 4 AMU were taken into account in this work, as the intensities are significantly higher there. At AUG, RGA data was acquired by several MSs, located in four diagnostic clusters. An MKS HPQ2 MS was connected to the inner divertor, an MKS HPQ3 and MicroVision 2 MSs were connected to the outer divertor and a Hiden Analytical HAL 201 RC MS was connected to the midplane. As the discharge duration at AUG is shorter than at JET (8 seconds vs. typically 25 seconds respectively), the scanning times of the RGAs were generally kept around $0.5 \mathrm{~s}$ to still record several scans per discharge. Each device could thus record either the hydrogen range or the 
impurity range, but not both. The hydrogen range was recorded in the inner and outer divertor (with the MKS HPQ2 at $1.2 \mathrm{~s}$ per scan and the MicroVison2 at $0.3 \mathrm{~s}$ per scan), and the impurities range was recorded in the outer divertor and in the midplane (with the MKS HPQ3 at $0.7 \mathrm{~s}$ per scan and the Hiden Analytical HAL $201 \mathrm{RC}$ at 0.36 s per scan).

In both fusion devices, the RGAs were calibrated for the cracking pattern of methane and hydrogen. At JET, the calibration was done exclusively in-situ, by injecting $\mathrm{D}_{2}$ and $\mathrm{CD}_{4}$ into the reactor vessel whereas at AUG the in-situ calibrations were complemented with laboratory calibration procedures. The uncertainty of the cracking patterns in both devices, for all MSs, was estimated at about $10 \%$. To analyse the local atmosphere at each RGA, and its potential impact on the measured ratios, the instruments were acquiring data continuously between discharges.

\section{Results and discussion}

The $\mathrm{H} /(\mathrm{H}+\mathrm{D})$ ratios of hydrogen containing molecules were evaluated with a statistical model which takes into account the cracking pattern of the expected molecules and its uncertainty $(9,10)$. The molecules, observed in this analysis were hydrogen and methane. In the general case, the model takes into account all of the possible isotope configurations of each specie. For hydrogen, these are: $\mathrm{D}_{2}, \mathrm{DH}$ and $\mathrm{H}_{2}$, and for methane they are: $\mathrm{CD}_{4}, \mathrm{CD}_{3} \mathrm{H}, \mathrm{CD}_{2} \mathrm{H}_{2}$, $\mathrm{CDH}_{3}, \mathrm{CH}_{4}$. The model assumes an average $\mathrm{H} /(\mathrm{H}+\mathrm{D})$ ratio for each specie, and the corresponding partial pressure is the sum of the partial pressures of all of the isotope configurations, weighted with the appropriate probability (calculated according to the binomial probability distribution function). The result of the fitting is then the partial pressure and average isotope ratio of hydrogen and methane. Beside the average-isotope molecules, isotopically pure molecules (such as $\mathrm{H}_{2}$ and $\mathrm{D}_{2}$ ) can also be used in the fit. This is of use in cases when a pure hydrogen isotope is injected into a reactor whose walls are loaded with the other isotope (e.g. $\mathrm{H}_{2}$ injected into a D-loaded machine) as it can help to assess the fuelling efficiency. The use of this type of evaluation is described in more detail in the discussion about results from AUG.

Hydrogen is the obvious choice for an observed molecule as it is injected as plasma fuel, retained and released(7) from the walls during plasma operation. On the other hand, methane is formed in through erosion of carbon from vessel surfaces. It has been identified as the most prominent impurity in non-seeded discharges, and least affected by the local atmosphere of the MSs(5). Although the carbon content in the plasmas has been significantly reduced in the transition from carbon-based to fully metallic plasma facing components (PFCs), it has nevertheless not been completely eliminated(11). The majority of the PFCs are not bulk metal, but metal-coated carbon-based materials (graphite and carbon fibre composites), so the substrate material close to the plasma-facing areas can still act as a source of carbon. Moreover, in extreme cases, degradation of the coatings can result in delamination, exposing the carbon substrate directly to the plasma. Further sources of carbon can be found in debris which accumulates on the vessel floor and amorphous carbon deposits, formed after the erosion of carbon. Other molecules formed from plasma species and impurity atoms have either not been identified, or were found unsuitable for assessment of the isotope ratio as they engage in isotope exchange in plasmashaded areas $(5,9)$. 
An example of discharge time traces of $\mathrm{H} /(\mathrm{H}+\mathrm{D})$ ratios from various diagnostics at JET, including methane and hydrogen from RGA, is shown in Fig 1 a, together with the (uncalibrated) partial pressures of hydrogen and methane (Fig 1 b), the plasma current (Fig 1 b) and gas injection (Fig $1 \mathrm{c}$ ). At the start of the pulse, the pressures of hydrogen and methane are too low to reliably detect the isotope ratio. Sufficient partial pressures are established at the onset of the divertor phase at $3.5 \mathrm{~s}$, as already shown in (5). At that point, signals from the divertor spectroscopy become available as well. Between the 6 and $7 \mathrm{~s}$ mark, significantly more $\mathrm{D}_{2}$ than $\mathrm{H}_{2}$ is injected, which is reflected in the spectroscopy and the Penning gauge signals. The hydrogen $\mathrm{H} /(\mathrm{H}+\mathrm{D})$ ratio in the RGA measurements follows this sudden dip, but not the methane $\mathrm{H} /(\mathrm{H}+\mathrm{D})$ ratio. Based on this variation, we estimate that the time resolution of the assessment of the isotope ration from the methane molecule is lower than 3 seconds, while the time resolution of the assessment of the isotope ratio from the hydrogen molecule is limited by the scanning rate of the RGA (1.3 s). After that, the injection of $\mathrm{H}_{2}$ and $\mathrm{D}_{2}$ is constant, and roughly the same, which also results in significantly smaller variations in all of the observed the isotope ratio signals. It is reassuring that the ratios from both RGA signals show very similar trends and absolute values as the ratio from the divertor spectroscopy. In contrast to the other signals, the ratios from RGA show considerable stability at the end of the pulse. This stability continues into the outgassing phase, unlike the ratio as deduced from the Penning gauge data, which could be explained by local differences in the pumping of each diagnostic.

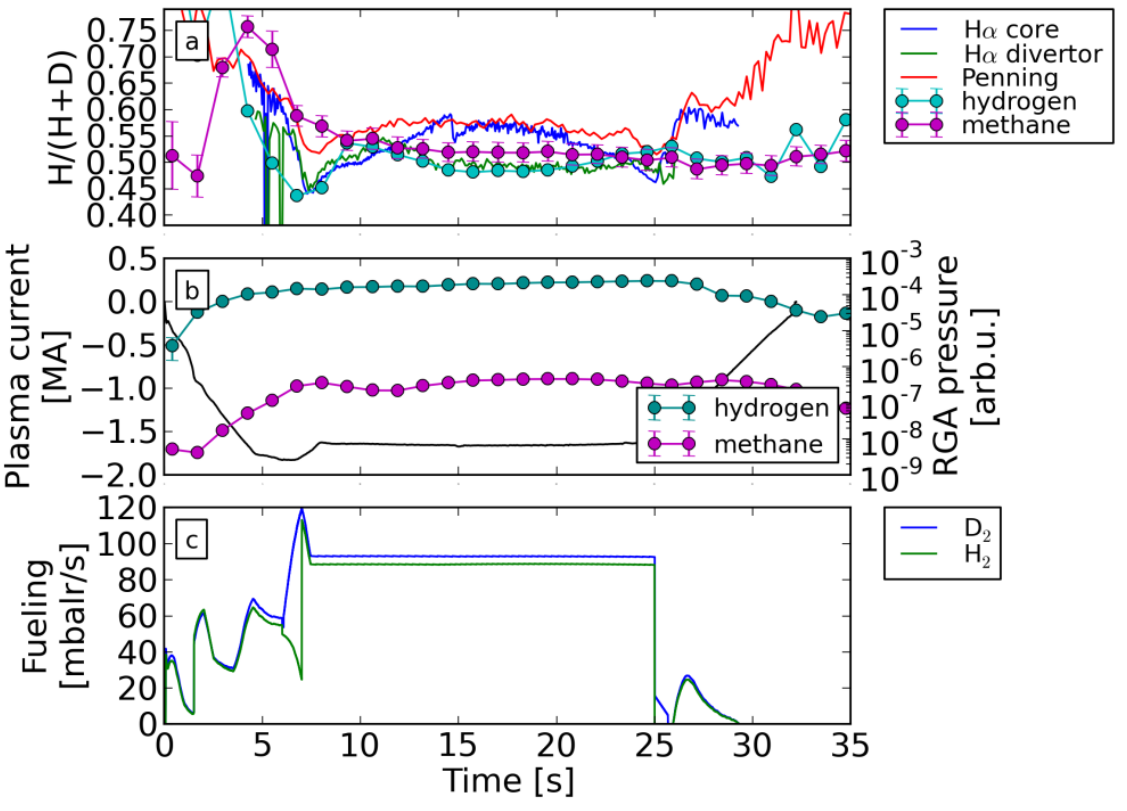

Figure 1. Time traces of the JET discharge 91016, showing H/(H+D) ratios from emission spectroscopy, Penning gauge spectroscopy and RGA (a), together with partial pressures of methane and hydrogen detected by RGA (b), plasma current (b) and $\mathrm{D}_{2}$ and $\mathrm{H}_{2}$ injection (c).

The validity of the ratios from RGA is further confirmed in Fig. 2, which shows the average isotope ratio in a 3 second period at the middle of the discharge. The data is plotted against the values of the core isotope ratio (as provided by the hydrogen alpha spectroscopy), as it is the most important one for the operation of the fusion device. For this evaluation, the discharge 
interval is defined as the interval where the plasma current is above $75 \%$ of its maximal value, and the methane partial pressure is sufficient for determination of the $\mathrm{H} /(\mathrm{H}+\mathrm{D})$ ratio. Generally, the isotope ratios from the hydrogen and methane molecules agree very well with the ratios from the other diagnostics. While the relatively wide error bars outline a certain naivety of the approach, the dispersion of the values of the RGA signals is not significantly greater than of other signals. Most notably, while the methane signal generally shows similar values as the divertor spectroscopy, it sometimes tends to show a lower $\mathrm{H} /(\mathrm{H}+\mathrm{D})$ ratio than the other signals. This could be interpreted by the fact that the methane is also formed through chemical erosion of D-rich carbon deposits in the plasma shaded areas, and does not re-enter the plasma. The deposits are then an additional source of $\mathrm{D}$ which is otherwise not accessible to the plasma and thus does not impact other signals of the isotope ratio.

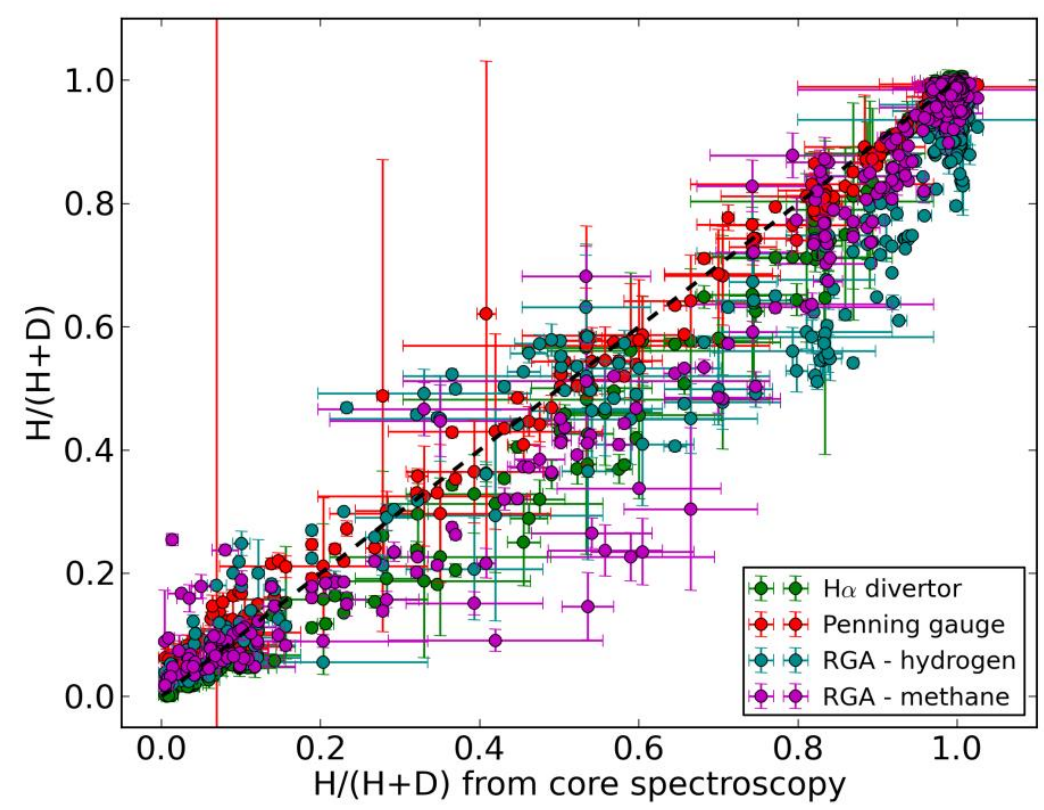

Figure 2. Average values of $\mathrm{H} /(\mathrm{H}+\mathrm{D})$ ratios from the sub-divertor Penning gauge, $\mathrm{H}$-alpha divertor spectroscopy and RGA hydrogen and methane signals in JET discharges

\section{AUG}

Time traces of HD ratios from various signals in an AUG discharge are shown in Fig. 3, together with the plasma current and $\mathrm{H}_{2}$ injection. The isotope ratios from the RGA data all indicate a higher D content that the NPA data, and within the RGA isotope ratios, there is also a considerable amount of dispersion. The reported values range from 0.2 to 0.45 before the onset of the $\mathrm{H}_{2}$ injection at $1.8 \mathrm{~s}$, and from 0.3 to 0.55 during the $\mathrm{H}_{2}$ injection. All of the ratios of from the RGAs are also considerably lower than the ratio reported by the NPA, which averages around 0.55 before and 0.68 after the start of the $\mathrm{H}_{2}$ injection. The general trends the signals exhibit are, however, very similar. As in the case of the JET data, the isotope ratio evaluated from the methane molecule shows a noticeable delay (roughly $1 \mathrm{~s}$ ) while the response of the isotope ratio 
of the hydrogen molecule is limited by the recording rate of the RGA. The similarity of the trends of the isotope ratios from all diagnostics can also be seen in Figs 4 and 5 which show the average value of the $\mathrm{H} /(\mathrm{H}+\mathrm{D})$ ratios in a fixed interval, plotted against the ratio as reported by NPA and shot number, respectively. While there is very noticeable scatter, especially in the midplane RGA data, generally the HD ratios from RGAs follow the trends of the NPA data, and consistently show a very pronounced spatial distribution of the HD ratio. The highest $\mathrm{H} /(\mathrm{H}+\mathrm{D})$ ratios are detected by the midplane RGA and the lowest are detected by the RGAs in the outer divertor.

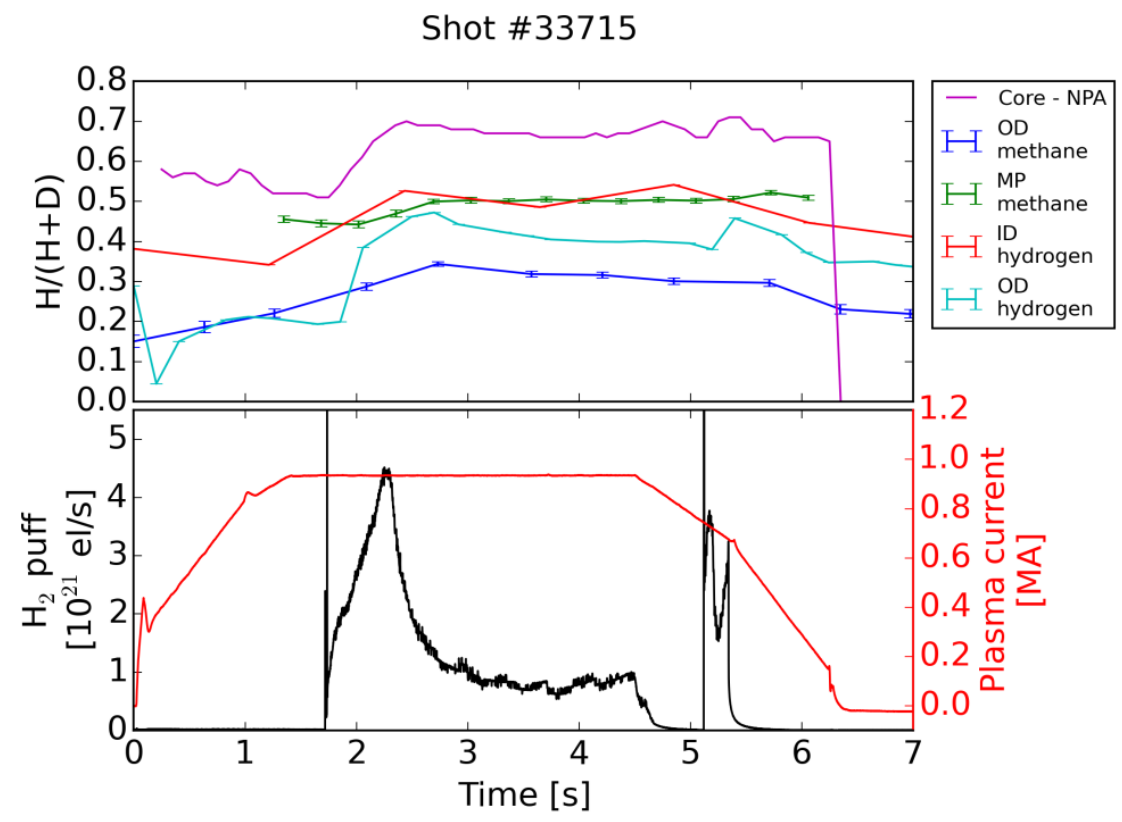

Figure 3. Time traces of the AUG discharge \#33715, showing $\mathrm{H} /(\mathrm{H}+\mathrm{D})$ ratios from the RGAs at AUG, together with the ratio from the NPA data, plasma current and $\mathrm{H}_{2}$ injection.

This indicates the impact of plasma-wall interaction and the recycling of fuel from the walls. In the inner divertor, where the pressure during the discharge is around an order of magnitude higher than in the outer divertor, the $\mathrm{D}$ released from the walls is diluted with $\mathrm{H}$ to a greater extent. As at JET, the methane $\mathrm{H} /(\mathrm{H}+\mathrm{D})$ ratio in the outer divertor is lower than the $\mathrm{H} /(\mathrm{H}+\mathrm{D})$ ratio of the hydrogen molecule which again might indicate that additional $\mathrm{D}$ reservoirs are being released from the carbon deposits in the plasma shaded areas. 


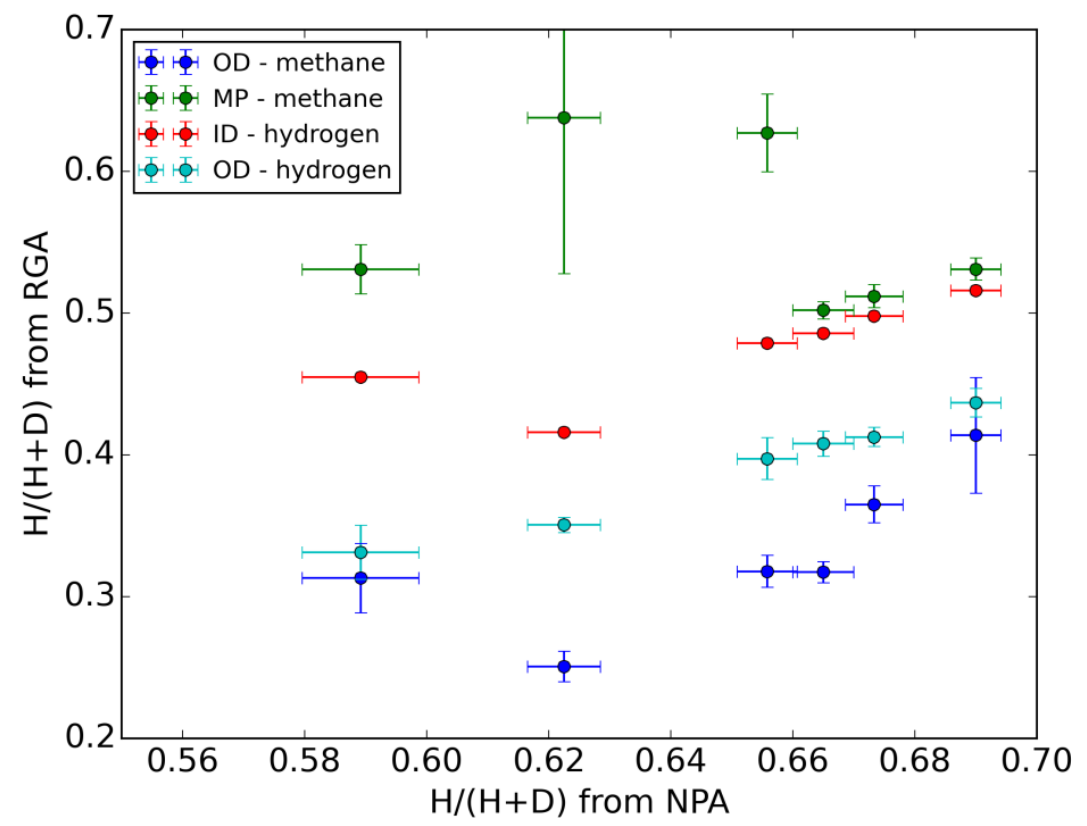

Figure 4. Average values of the $\mathrm{H} /(\mathrm{H}+\mathrm{D})$ ratios from the RGAs at $\mathrm{AUG}$, plotted against the average value of the $\mathrm{H} /(\mathrm{H}+\mathrm{D})$ ratio from the NPA data, all from the $3.2-4.4 \mathrm{~s}$ interval.

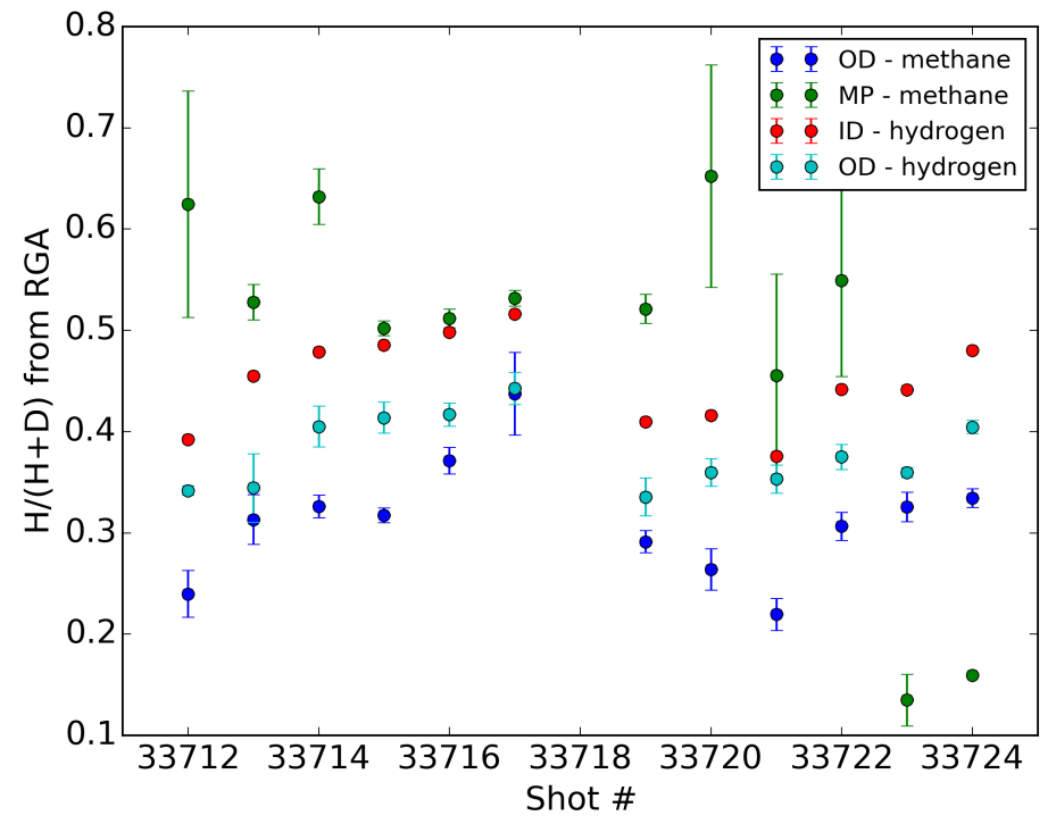

Figure 5. Average values of the $\mathrm{H} /(\mathrm{H}+\mathrm{D})$ ratios from the RGAs at AUG from the $2.8-4.4 \mathrm{~s}$ interval, plotted against shot number.

The discharges of the hydrogen campaign at AUG were performed with pure $\mathrm{H}_{2}$ fuelling. In the first discharges in this series, it is thus reasonable to expect that the wall is still predominantly loaded with deuterium. Then, it is possible to evaluate the degree of the dissociation of the 
injected gas from the $\mathrm{H} /(\mathrm{H}+\mathrm{D})$ ratio of the exhaust gas. This is done by fitting the RGA data with two separate hydrogen species: $\mathrm{H}_{2}$ - a fully protonated molecule and hydrogen - a mixed isotope molecule (whose isotope ratio is varied in the fit). The plasma fuel consists of the injected and dissociated $\mathrm{H}_{2}$ as well as the $\mathrm{D}$ atoms ejected from the walls. The hydrogen molecules formed through recombination of plasma radicals have the average isotope ratio of the plasma hydrogen content. On the other hand, in the fraction of injected $\mathrm{H}_{2}$ that does not reach the plasma, the isotope ratio of the molecules does not change. Indeed, as shown in Fig 6, the time trace of the partial pressure of the non-dissociated $\mathrm{H}_{2}$ largely resembles the time trace of the absolute neutral gas pressure (Fig 6 a) whereas the time trace of the partial pressure of the mixed-isotope hydrogen molecule resembles the time trace of the core electron density (Fig $6 \mathrm{~b}$ ). The neutral gas pressure shows a significant increase with the onset of fuelling (Fig 6 e), while rise of the electron density is considerably slower. For this comparison, the data from the outer divertor RGA is used, as it features the highest time resolution, although the same trends can be observed with the outer divertor RGA as well. Namely, the isotope ratio of the mixed isotope hydrogen molecule (Fig $6 \mathrm{c}$ ) and the evaluated degree of dissociation (Fig $6 \mathrm{~d}$ ) are similar in both regions.

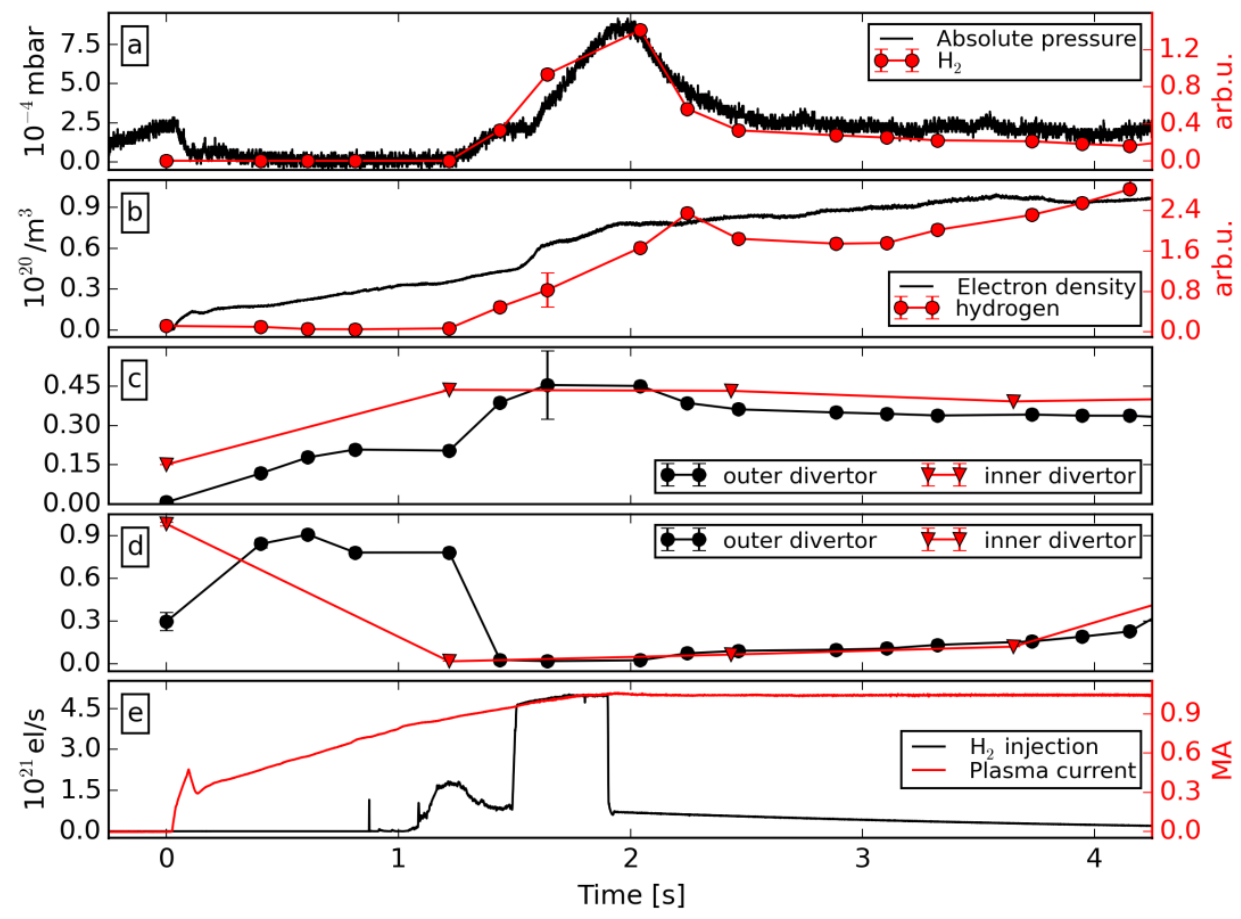

Figure 6. Time traces of the AUG discharge \#33712, showing the divertor absolute pressure and the outer divertor fitted pure $\mathrm{H}_{2}$ (a), core electron density and mixed-isotope hydrogen partial pressures in the outer divertor(b), together with the $\mathrm{H} /(\mathrm{H}+\mathrm{D})$ ratio of the mixed-isotope hydrogen (c) and the degree of dissociation (d) from both RGAs, and the $\mathrm{H}_{2}$ injection and plasma current (e)

\section{Conclusions}


The presented results clearly illustrate that RGA can be indeed used to monitor the isotope content in the plasma. Especially at JET, where the database of signals comprises data from 433 pulses, the isotope ratios of both the hydrogen and the methane molecules generally agree with the ratios detected by other diagnostics, however with a considerable scatter. While the detected discrepancies could be partially attributed to the limited temporal resolution of the method, especially for the methane signals, they could as well reflect real differences in the isotope ratios measured by different diagnostics at different locations, among which especially the methane molecule might be produced also from the a-C:D deposits in plasma shaded areas which are otherwise not accessible to the plasma. The spatial distribution is even more evident at AUG, where the RGA set up allows for a consistent and clear observation of a spatial distribution of the measured isotope ratios. As the isotope ratios measured by RGA are consistent with other diagnostics, they can provide insight into the dynamics of isotope changeover experiments, and can be also used to evaluate the fuelling efficiency.

The agreement of the RGA data with the existing diagnostics shows that RGA can be effectively used to monitor the isotope ratios of the plasma in fusion devices, which in turn can help to characterize wall conditioning processes or the plasma fuel mixture in the active phase of operations. While in future fusion devices the diagnostic possibilities will be more severely limited than in today's experimental reactors, RGA will still remain a viable diagnostic as it does not require direct contact or close vicinity to the plasma, and thus will be in the position to provide key information of the plasma wall processes in the reactor chamber, and the overall performance of the fusion device.

In the presented data, the time resolution of the isotope ratio as determined from the methane molecule was between 1 and $3 \mathrm{~s}$ whereas the time resolution of the isotope ratio of the hydrogen molecule was limited by the RGA recording rate $(0.3 \mathrm{~s})$. The present time resolution is likely not high enough for use in real-time control systems, however the true time resolution of the method will still have to be evaluated in future experiments.

\section{Acknowledgements}

This work has been carried out within the framework of the EUROfusion Consortium and has received funding from the Euratom research and training programme 2014-2018 under grant agreement No 633053. The views and opinions expressed herein do not necessarily reflect those of the European Commission.

\section{References}

1. Loarer T, Corre Y, Delpech L, Devynck P, Douai D, Ekedahl A, et al. Fuel recovery experiments with isotopic plasma wall changeover during long discharges in Tore Supra. Nucl FUSION. 2013 Mar;53(3).

2. Hillis DL, Hogan J, Zastrow K-D, Parail V, Coster D, Reiter D, et al. Role of recycling in JET trace tritium transport experiments. J Nucl Mater. 2007 Jun 15;363:498-504.

3. Klepper C, Hillis D, Wade M, Maingi R, McKee G. Application of a species-selective Penning gauge to the measurement of neon and hydrogen-isotope partial pressures in the plasma boundary. Rev Sci Instrum. 1997 Jan;68(1, 2):400-3. 
4. BARTIROMO R, BRACCO G, BRUSATI M, GROSSO G, MANTOVANI S, TILIA B, et al. DESIGN AND CALIBRATION OF THE JET NEUTRAL PARTICLE ANALYZER. Rev Sci Instrum. 1987 May;58(5):788-95.

5. Drenik A, Oberkofler M, Alegre D, Kruezi U, Brezinsek S, Mozetic M, et al. Mass spectrometry analysis of the impurity content in N2 seeded discharges in JET-ILW. J Nucl Mater. 2015;463:684-687.

6. M Oberkofler and G Meisl and A Hakola and A Drenik and D Alegre and S Brezinsek and R Craven and T Dittmar and T Keenan and S G Romanelli and R Smith and D Douai and A Herrmann and K Krieger and U Kruezi and G Liang and Ch Linsmeier and M Mozetic and V Rohde and the ASDEX Upgrade team and the EUROfusion MST1 Team and JET Contributors. Nitrogen retention mechanisms in tokamaks with beryllium and tungsten plasma-facing surfaces. Phys Scr. 2016;2016(T167):014077.

7. Brezinsek S, Loarer T, Philipps V, Esser HG, Grunhagen S, Smith R, et al. Fuel retention studies with the ITER-Like Wall in JET. Nucl Fusion. 2013 Aug;53.

8. Kruezi U, Sergienko G, Morgan PD, Matthews GF, Brezinsek S, Vartanian S, et al. JET divertor diagnostic upgrade for neutral gas analysis. Rev Sci Instrum. 2012 Oct;83.

9. Neuwirth D, Rohde V, Schwarz-Selinger T, Asdex Upgrade Team. Formation of ammonia during nitrogen-seeded discharges at ASDEX Upgrade. Plasma Phys Control Fusion. 2012 Aug;54.

10. Drenik A, Alegre D, Brezinsek S, Castro A de, Kruezi U, Meisl G, et al. Detection of ammonia by residual gas analysis in AUG and JET. Fusion Eng Des [Internet]. 2017 May 31; Available from: http://www.sciencedirect.com/science/article/pii/S0920379617305811

11. Brezinsek S, Jachmich S, Stamp MF, Meigs AG, Coenen JW, Krieger K, et al. Residual carbon content in the initial ITER-Like Wall experiments at JET. J Nucl Mater. 2013 Jul;438:S303-8. 